Acta Crystallographica Section D

Biological

Crystallography

ISSN 0907-4449

Thomas S. Walter, Jonathan M. Diprose, Chris J. Mayo, Christian Siebold, Mike G. Pickford, Lester Carter, Geoff C. Sutton, Nick S. Berrow, James Brown, Ian $M$. Berry, Guillaume B. E. StewartJones, Jonathan M. Grimes, David K. Stammers, Robert $M$. Esnouf, E. Yvonne Jones, Ray J. Owens, David I. Stuart and Karl Harlos*

Oxford Protein Production Facility, Division of Structural Biology, Henry Wellcome Building for Genomic Medicine, Roosevelt Drive, Headington, Oxford OX3 7BN, England

Correspondence e-mail: karl@strubi.ox.ac.uk

(C) 2005 International Union of Crystallography Printed in Denmark - all rights reserved

\section{A procedure for setting up high-throughput nanolitre crystallization experiments. Crystallization workflow for initial screening, automated storage, imaging and optimization}

Crystallization trials at the Division of Structural Biology in Oxford are now almost exclusively carried out using a high-throughput workflow implemented in the Oxford Protein Production Facility. Initial crystallization screening is based on nanolitre-scale sitting-drop vapour-diffusion experiments (typically $100 \mathrm{nl}$ of protein plus $100 \mathrm{nl}$ of reservoir solution per droplet) which use standard crystallization screening kits and 96-well crystallization plates. For $294 \mathrm{~K}$ crystallization trials the barcoded crystallization plates are entered into an automated storage system with a fully integrated imaging system. These plates are imaged in accordance with a pre-programmed schedule and the resulting digital data for each droplet are harvested into a laboratory informationmanagement system (LIMS), scored by crystal recognition software and displayed for user analysis via a web-based interface. Currently, storage for trials at $277 \mathrm{~K}$ is not automated and for imaging the crystallization plates are fed by hand into an imaging system from which the data enter the LIMS. The workflow includes two procedures for nanolitre-scale optimization of crystallization conditions: (i) a protocol for variation of $\mathrm{pH}$, reservoir dilution and protein:reservoir ratio and (ii) an additive screen. Experience based on 592 crystallization projects is reported.

\section{Introduction}

In the last few years new instrumentation for liquid dispensing (Rose, 1999; Mueller et al., 2001; Bodenstaff et al., 2002; Krupka et al., 2002; Santarsiero et al., 2002; Shvets et al., 2002; Walter et al., 2003), automated storage and high-quality optical imaging (Adams et al., 2002; Kuhn et al., 2002) have provided opportunities to revolutionize procedures for protein crystallization experiments. Similarly, the application of a fully developed laboratory information-management system (LIMS) based approach to the organization and storage of crystallization trial data and images has been made practical by the provision of barcoding on standard plasticware and digitized imaging. In addition to the potential benefits of efficiency and reproducibility, one major advantage of the new hardware is that nanolitre-scale liquid dispensing allows a reduction by one or more orders of magnitude in the amount of protein required for initial crystallization screening experiments. Meanwhile, LIMS databases facilitate the development of software for crystal image recognition, the systematic analysis of time courses for crystal growth (Spraggon et al., 2002; Wilson, 2002, 2004; Mayo et al., 2005) and data mining for the refinement of crystallization screens. The development of protocols and workflows which exploit the advances in hardware and software technologies has in large part been driven by the challenges of constructing 'high-throughput' structural biology or 'structural genomics' pipelines. However, many of the procedures and much of the instrumentation implemented in such facilities are now recognized to be of equal applicability and benefit to 'classical' protein crystallographic laboratories.

We present here an overview of the current workflow for crystallization experiments implemented in the Oxford Protein Production Facility (OPPF). The OPPF is funded by the UK Medical Research Council as a pilot project to develop and apply high-throughput methodologies to the structural biology of biomedically relevant proteins. The crystallization workflow is routinely used for virtually 
all the proteins (and protein complexes) entering protein crystallographic studies within the Division of Structural Biology of the Department of Medicine. To date, this numbers 592 projects. We have previously reported the design and validation of a protocol for setting up nanolitre-scale sitting-drop vapour-diffusion experiments (Walter et al., 2003). At the same time we reported an initial tranche of crystallization results based on the first 50 projects to make day-today use of the liquid-dispensing instrumentation (Brown et al., 2003). The protocols for setting up nanolitre-scale crystallization trials have now been expanded to include basic procedures for crystal optimization and the workflow extended to encompass automated storage and imaging. Crystallization drop data and digitized images are captured in a LIMS which is accessed via a web-based interface incorporating tools for image display and analysis (Mayo et al., 2005). The LIMS database is underpinning the development of crystal recognition software and already provides a potentially significant resource for data mining to refine crystallization strategies.

\section{Experimental}

\subsection{Crystallization plates and screening kits}

96-well crystallization plates with square protein wells and flatbottom platforms are supplied barcoded from Greiner (reference No. 609.101, Greiner Bio-One Ltd, Stonehouse, UK) and manually sealed for crystallization trials using transparent self-adhesive foil (Viewseal, Greiner). Reagents from commercially available crystallization screening kits (Hampton Research, CA, USA and Emerald BioStructures, deCode Genetics, WA, USA) are reformatted (using a Qiagen BioRobot 8000, Qiagen Ltd, Crawley, UK) into sets of 96 conditions in deep-well blocks ( $2 \mathrm{ml}$ Masterblock-PP, Greiner). Five blocks are used to provide a standard set of 480 conditions, namely: (i) Hampton Screens I and II, (ii) Wizard Screens I and II, (iii) PEG/ Ion, Grid Screens PEG 6000 and AS (Hampton), (iv) Natrix and Cryo Screens (Hampton), (v) Grid Screens PEG/Li, NaCl, MPD and 'Quik Screen' Phosphate (Hampton).

These blocks were established at the start of the high-throughput project and were deliberately based on the commercial kits then favoured within our standard crystallization laboratory. We were aware that this would lead to some duplication of conditions; however, we wished initially to stick to well established reagents with the intention of building up a sufficiently large database to allow a statistical analysis of success rates to redefine the panel of conditions at a later stage. This strategy would also allow direct comparison, from the outset, between hand- and robot-dispensed crystallization drops.

After the general efficacy of the nanolitre-scale protocol had been demonstrated (Brown et al., 2003) we introduced further blocks. The first two blocks were newly introduced commercial screens, namely Index (Hampton) and SaltRx (Hampton). These were made available to strengthen our intended statistical analysis. The final two blocks were devised to deal with special cases. The first of these was designed to address the situation where only a very small amount of protein was available. The 96 conditions were chosen by simple selection of the 48 'best' conditions from Hampton Screens I and II ('best' as judged by us from data on crystallization success rates provided by Hampton Research) together with 48 Hampton PEG/Ion conditions, which we observed to be performing strongly from our own initial results. Finally, a focused TCR-MHC block was designed based on our in-house experience and a survey of published crystallization conditions for these molecules, this block is strongly biased towards
PEG-based conditions (Stewart-Jones et al., manuscript in preparation).

Storage blocks are manually sealed with metal sealing foil (ABGene, Adhesive PCR Sealing Foil, Product Code AB-0626) and are typically kept for up to three weeks at room temperature. Blocks which are not required within this time are stored at $277 \mathrm{~K}$.

\subsection{Pipetting hardware and protocols}

Where possible proteins are concentrated to $10 \mathrm{mg} \mathrm{ml}^{-1}$ and subjected to a pre-crystallization test ('PCT', Hampton) to check that this concentration is suitable prior to committing the bulk of the sample to a full crystallization screen. The standard OPPF procedure for set-up of a 96-well crystallization plate remains that described by Walter et al. (2003), in brief: (i) dispense precipitant solutions into Greiner plate reservoirs (using a Robbins Hydra; Matrix Technologies Ltd, Wilmslow, UK), (ii) pipette $96100 \mathrm{nl}$ protein droplets using a single tip of the Cartesian Technologies Microsys MIC4000 and (iii) add a $100 \mathrm{nl}$ droplet of reservoir to each crystallization platform using the Cartesian instrument in single-dispense mode (reservoir solutions corresponding to a column of the 96-well plate being pipetted simultaneously by the eight tips). Start-up and shut-down programmes to clean the Cartesean tips and remove any air-bubbles are run on a daily basis and tip-cleaning as well as machine-maintenance procedures are carried out once a week. All pipetting operations are carried out at $294 \mathrm{~K}$. For crystallization trials at $277 \mathrm{~K}$ the plates are then placed in a thermally insulated box and transferred to $277 \mathrm{~K}$. In this way the temperature is decreased gradually over a period of $24 \mathrm{~h}$, avoiding significant condensation of reservoir solution onto the sealing foil.

\subsection{Automated crystallization storage and imaging hardware}

For experiments at $294 \mathrm{~K}$, crystallization plates are manually fed into an automated storage vault designed and manufactured by The Automation Partnership (TAP; Royston, UK) to meet the following specifications: storage capacity of 10000 crystallization plates, maximum temperature variation anywhere in the store $\pm 1.0 \mathrm{~K}$. Robot acceleration/deceleration rates were reduced to avoid abrupt plate movements and minimize vibrations transmitted to the stored plates. The storage vault was modified by TAP to incorporate an annexe which houses an Oasis 1700 imaging system (Veeco, Cambridge, UK). Additional pick-and-place robots, integrated with the storage vault, transfer crystallization plates to and from the imaging system, allowing routine imaging without manual intervention. Both the storage vault and imaging system are controlled by PCs with exchange of data between them achieved via a custom DCOM interface. Details of this aspect of the workflow are described by Mayo et al. (2005). Images are transferred to a large central storage system by gigabit ethernet. A stereo-microscope (SMZ1500, Nikon Ltd, Kingston, UK) is available to look at individual plates in more detail. High-resolution colour images can be taken with a digital camera (Pixera 120es, Digital Imaging Systems Ltd, Egham, UK) and these images are integrated into the central image storage system.

For experiments at $277 \mathrm{~K}$, crystallization plates are stored in a home-made racking system within a standard cold room. Imaging is carried out by manually loading cassettes containing up to 32 plates into an Oasis 1750 imaging system (Veeco, Cambridge, UK) positioned next to the racking system. The imaging system is controlled by a PC which transfers images into the unified central storage system using fast ethernet. 


\subsection{Computer control, LIMS and image analysis}

Crystallization plates are supplied pre-barcoded using Code128 symbology. Plate barcodes are scanned using a DataLogic Gryphon D100 handheld barcode reader (DataLogic UK Ltd, Redbourne, UK) when the reservoir wells are filled, creating an entry in the protein production LIMS (unpublished implementation by JMD in Nautilus, ThermoInformatics, Altrincham, UK). As the crystallization trials are set up, the barcode is checked again using a PSC LM520 barcode reader (PSC Bar Code Ltd, Watford, UK) mounted on the Cartesian Microsys MIC4000. On reception of a plate into the storage vault, a record and imaging schedule are created in the storage vault database. Imaging occurs as soon as possible after each scheduled time and $1024 \times 1024$ eight-bit bitmap (.BMP) well images are cropped (to $750 \times 700$ ), named with the barcode, well name, time and date and transferred to the central storage system. Images are stored on a 2 terabyte IDE-SCSI ADV RAID (JAD Logic, Plymouth, UK) and analysed automatically on a Linux farm comprising three dual-processor nodes (Dell Computer, Bracknell, UK) using software developed at York University (Wilson, 2002, 2004). The images and the results of the image analysis are accessable to users via a web interface (described in detail in Mayo et al., 2005). In addition to the automatic image analysis the web interface allows users to annotate images manually and this annotation currently forms the basis of our statistical analyses of success rates. Imaging results and the web interface are controlled by PlateDB software, developed at the OPPF (Mayo et al., 2005).

\subsection{Standardized procedures for crystallization optimization}

The basic optimization and additive protocols use the reagents and instrumentation as detailed above but with the following modifications.

(i) In the basic optimization protocol only rows $\mathrm{F}, \mathrm{G}$ and $\mathrm{H}$ of the Greiner plate are used (i.e. 36 wells). For an initial crystallization condition of $\mathrm{pH} X$ stock solutions of approx. $\mathrm{pH} X-0.5$ and $X+0.5$ are generated by hand (by addition of small volumes of $\mathrm{HCl}$ and $\mathrm{NaOH}$, respectively, predetermined by empirical titration and generally in the range of 5-30 $\mu \mathrm{l}$ per $1 \mathrm{ml}$ of stock solution). These solutions are pipetted as dilution series [either manually or using a programmed protocol implemented on an MWG RoboGo, MWG Biotech (UK) Ltd, Milton Keynes, UK] into the appropriate Greiner plate reservoirs. Nanolitre-scale sitting drops with a range of protein to reservoir solution ratios, to effectively vary the protein concentration in the final drop, are then set up using a programmed protocol implemented on the Cartesian Technologies Microsys MIC4000.

(ii) For the additive protocol the 72 reagents of Hampton Additive Screens 1, 2 and 3 are divided into 56 non-volatile and 16 volatile reagents and reformatted into a 96well plate, occupying $9 \times 8$ columns. The original Hampton formulation of nonvolatiles is diluted 5:1 with water. The transfer of additive reagents to the crystallization plate is carried out with the Cartesian as an extra step to the normal procedure using all eight tips in parallel with the additive plate placed in one of the normally unused positions of the Cartesian bed. The non-volatile reagents are dispensed as additional $100 \mathrm{nl}$ aliquots directly into the crystallization droplets, whereas for the volatile reagents an aliquot of $20 \mu \mathrm{l}$ is dispensed into the reservoir solutions (after the $100 \mathrm{nl}$ transfer of reservoir solution to

Figure 1

Schematic diagram of the crystallization workflow at the Oxford Protein Production Facility starting with a small aliquot of concentrated protein (DLS, dynamic light scattering). The brain indicates a decision point for the user.
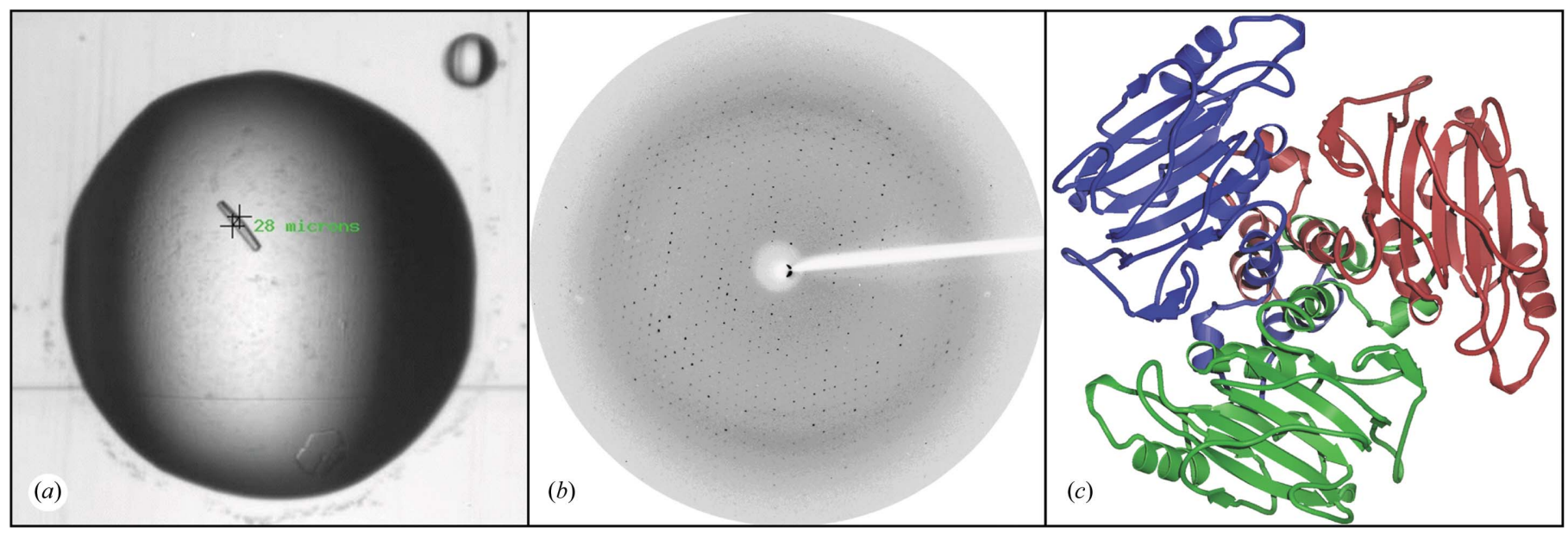

Figure 2

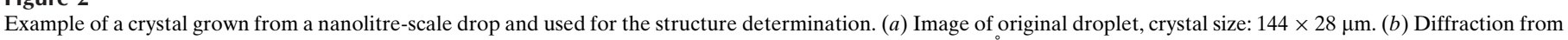

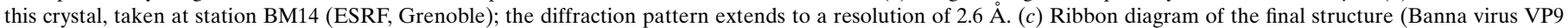
protein; Jaafar et al., 2005). 
the droplet). The anti-evaporation cover (a modification of the Cartesian described in Walter et al., 2003) has been extended to ensure that the additive plate is also fully covered.

\section{Results and discussion}

A schematic representation of the current OPPF crystallization workflow is presented in Fig. 1. The workflow design is deliberately modular. Whilst this precludes full automation, it allows simple replication of units to overcome bottlenecks (e.g. the nanolitre pipetting) and allows flexibility in the addition or substitution of modules (e.g. optimization protocol).

\subsection{Nanolitre-scale crystallization screening}

Pre-crystallization quality-assurance tests of proteins include mass spectrometry (Q-Tof micro, Waters, Milford, MA, USA) and dynamic light scattering (Dyna Pro, Proterion, Piscataway, NJ, USA). For our standard drop volume a 96-well crystallization plate uses $15 \mu \mathrm{l}$ of protein solution (this includes the extra volume required to aspirate the protein sample into the dispensing tip). Thus, a test of the 480 conditions covered by the five basic screening blocks requires only $0.75 \mathrm{mg}$ of protein (assuming a concentration of $10 \mathrm{mg} \mathrm{ml}^{-1}$ ). $1 \mathrm{~h}$ $15 \mathrm{~min}$ is required to set up the five 96-well plates. This gives a throughput of about six proteins per day (or 30 full plates) per Microsys MIC4000. In our hands this instrument has proved to be robust and reliable over a period of some 30 months despite a heavy workload. In Brown et al. (2003) we reported preliminary data that pointed to an encouragingly high success rate for crystal growth in the nanolitre-scale screens. This has been borne out by our subsequent experience; in many cases, the definitive X-ray diffraction data set has been collected from crystals grown in $100 \mathrm{nl}$ plus $100 \mathrm{nl}$ drops (for an example, see Fig. 2). Virtually all initial crystallization trials in the Division of Structural Biology now use the nanolitre-scale protocols; as mentioned above this amounts to 592 projects to date. The result of this wholesale shift in crystallization strategy has been to generate a bottleneck in access to the pipetting hardware; to address this, we have recently installed a second Cartesian Technologies instrument. Our current best estimate of the cost per plate for a crystallization trial set-up and monitored in this facility is approximately $£ 50$ (based on dedicated manpower costs, equipment depreciation, maintenance, plasticware and reagents).

A comparison of the success rate of the different screening blocks carried out using the database of user-annotated drops is shown in Fig. 3. The number of wells per plate containing crystals is considerably higher for the limited protein and the TCRMHC blocks than any of the other blocks (thus for the TCR-MHC block the success rate is over 3.5 times the overall success rate for the generic blocks). This supports the approach of using a subset of the most promising conditions if only a limited amount of sample material is available and provides encouraging evidence that tailormade screens for specific classes of proteins can be very effective (in line with the findings reviewed by Page \& Stevens, 2004). As yet we have not carried out any finer level statistical analyses.

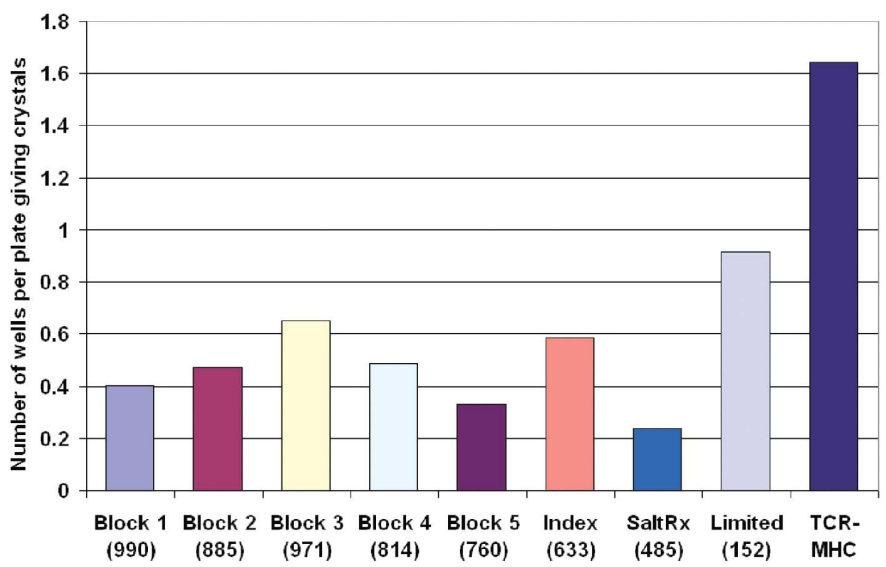

Figure 3

Success rate of the various screening blocks at $294 \mathrm{~K}$. Each block contains 96 reagents; block details are given in the text. Drops containing crystals were annotated manually. The number of plates set up using each block is given in parentheses.
Reservoir dilutions:

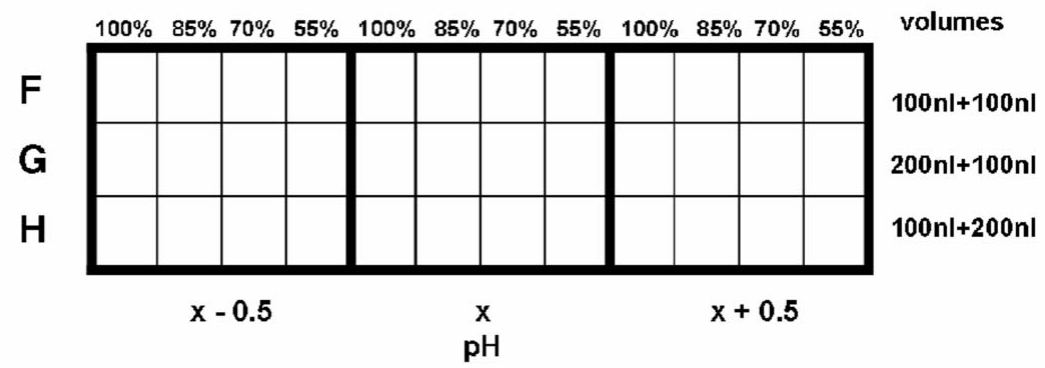

(a)

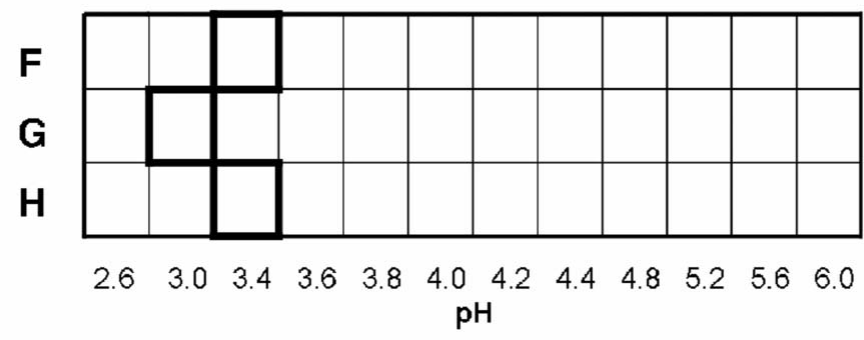

$100 \mathrm{nl}+100 \mathrm{nl}$

$200 \mathrm{nl}+100 \mathrm{nl}$

$100 \mathrm{nl}+200 \mathrm{nl}$
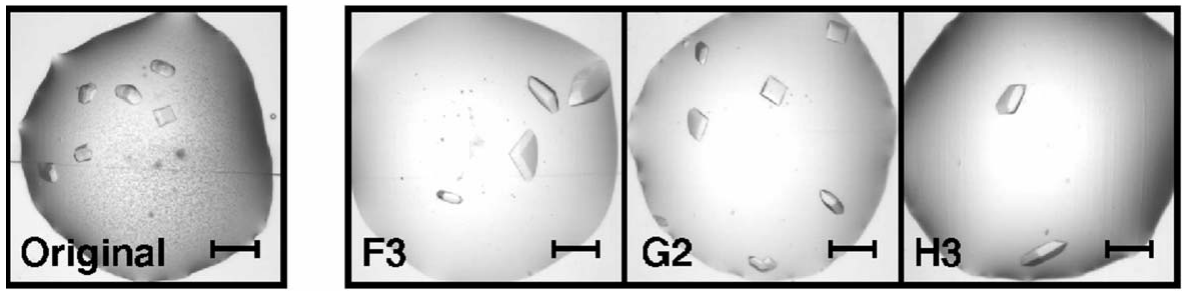

(b)

Figure 4

Optimization procedures using three rows of the 96-well plate. (a) Basic procedure varying the $\mathrm{pH}$, reservoir concentration and protein/reservoir ratio, $(b)$ specific optimization for SARS coronavirus nsp9 protein (OPPF 406). The original crystals were obtained from block 2 , solution $\mathrm{E} 9\left[2 M\left(\mathrm{NH}_{4}\right)_{2} \mathrm{SO}_{4}, 0.1 M\right.$ citrate-phosphate $\mathrm{pH}$ 4.2]. The structure was determined using the crystals from wells F3, G2 and H3 (Sutton et al., 2004). The scale bar represents $200 \mu \mathrm{m}$. 


\section{conference papers}

\subsection{Temperature as a variable}

We recognize that temperature is a potentially important variable for crystallization screening and have therefore set up smaller scale facilities at $277 \mathrm{~K}$. At present we endeavour, where sufficient protein is available, to set up crystallizations at both 277 and $294 \mathrm{~K}$ although in the case of shortage of material only $294 \mathrm{~K}$ is set up. To date 198 proteins have been set up at both 277 and $294 \mathrm{~K}$ and of these six have crystallized only at $277 \mathrm{~K}$. Interestingly, although in the vast majority of cases (147), crystals are obtained at both temperatures there is often a significant difference in the crystallization condition at the two temperatures. Further analysis of the efficacy of $277 \mathrm{~K}$ crystallization is ongoing.

\subsection{Nanolitre-scale crystallization optimization}

To date, two extra procedures have been incorporated into the OPPF crystallization workflow to attempt to address the often problematic issue of crystal optimization (alternative strategies have been proposed by Sulzenbacher et al., 2002 and Lartigue $e t$
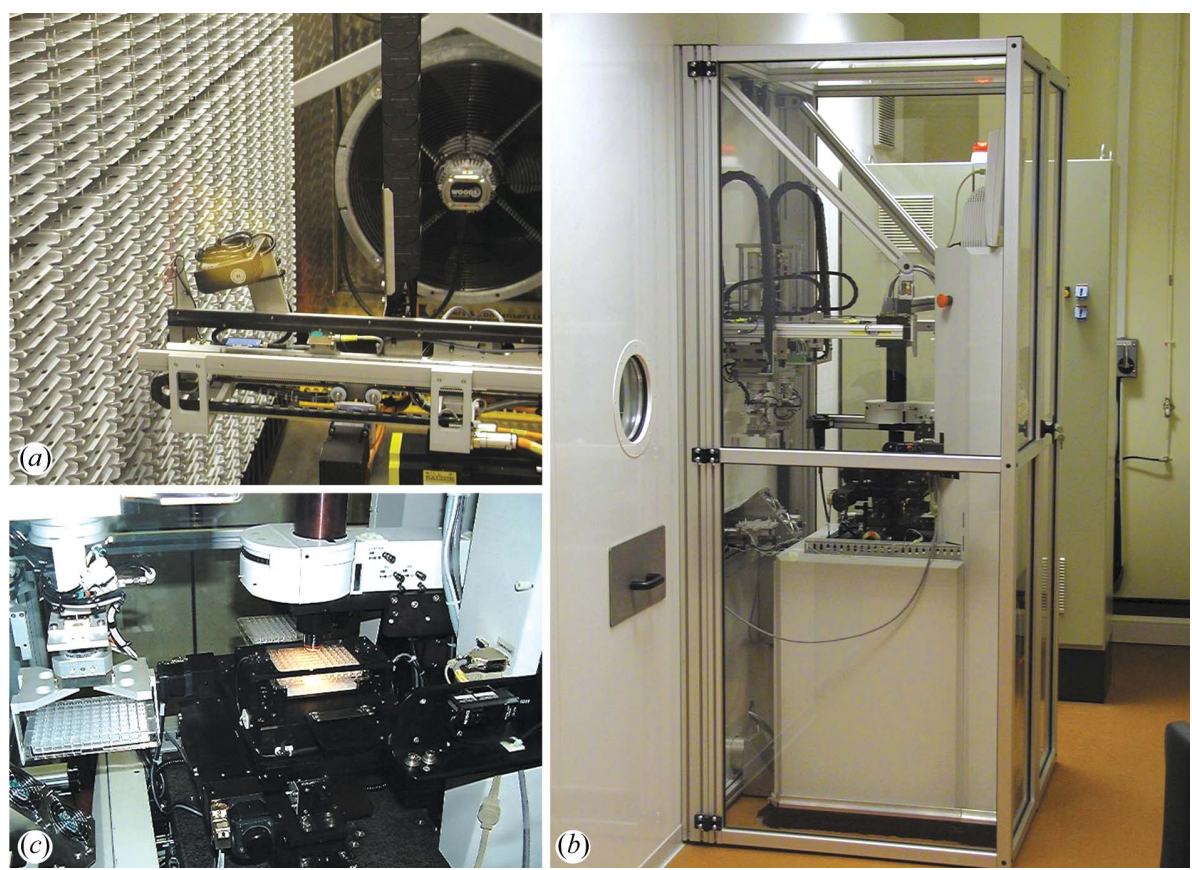

Figure 5

Automated storage and imaging. (a) View inside storage vault with racking system and tray transfer robot, $(b)$ imaging system as attached to the store, $(c)$ close up of the imaging system with one plate (in the centre) being imaged, a second plate (seen at the back) waiting to be imaged and a third plate (front, left) being transferred back into the store. al., 2003). The basic optimization procedure is detailed in Fig. 4(a). This mirrors well established strategies for standard sitting-drop vapour-diffusion experiments in that it varies $\mathrm{pH}$, reservoir dilution and protein concentration. These variables are equally relevant in nanolitre-scale crystallization and the protocol has yielded significant successes with many projects. The most arduous aspect of developing this protocol has been the requirement to determine $\mathrm{pH}$ titration values, to shift the $\mathrm{pH}$ by +0.5 and -0.5 , for each of the 480 conditions of our standard crystallization screen. In specific cases variations of this basic procedure may be more appropriate. Fig. 4(b) shows an example where the $\mathrm{pH}$ was varied over a broader range, but without a dilution series of the reservoir.

The second procedure, the additive screen, is again an adaptation to automated nanolitre-scale dispensing of a well established crystallization strategy. In this protocol the additive reagents are transferred with the Microsys MIC4000 as an extra step to the normal procedure as described above.

\subsection{Automated storage and imaging}

The majority of crystallization experiments carried out in the OPPF facility are maintained at $294 \mathrm{~K}$ in the automated storage vault (Fig. 5) described in $\$ 2.3$ (storage capacity 10000 Greiner plates). At the time of writing there are 6657 plates in the vault. All plate movements are controlled by the storage vault database and requests can be initiated via a user interface developed in-house. Plates are imaged on first entering the vault (defining the status of the drops at the start point of the crystallization experiment) and then at regular intervals (a total of 30 times over $315 \mathrm{~d}$ with shorter time intervals, $5 \mathrm{~h}$ at the start of the experiment lengthening to several days). The imaging speed is around one plate per minute with separate images taken for each well of the plate. Because of the small drop size the depth of focus is sufficient to cover the entire drop in a single image.

\subsection{Image display and analysis}

Individual images are classified using the York University software (Wilson, 2002, 2004), which is fully integrated with the crystallization control system. An image can be classified in $\sim 2-5$ s so that classification of images keeps pace with acquisition. Images are given a score of either -1 (unable to classify) or a number from 0 to 6 (varying from classification as a clear drop via different levels of precipitate and micro-crystals to single crystals). The accuracy of the current classification software is such that low scores (less than 2) are unlikely to be generated for images containing good crystals, but is not yet sufficient to replace manual inspection using the web interface.

A web interface gives a fast simple view onto the image database; this is described in detail in Mayo et al. (2005). The key features are displayed in Fig. 6. A list of plates can be browsed and an individual imaging session selected. The first view on an imaging session for a plate is a montage of the individual well images (Fig. 6d), with the colour of border around each image determined by its automatic classification score. Clicking on a well image produces the individual well view (Fig. 6e). For manual inspection of images a slide show mode can be selected. The order of images can either be determined by position on the plate or by the automatic classification score. Images can also be zoomed and objects measured. If examples of salt crystals for specific conditions have been previously identified these can be brought up in a separate window (Fig. 6b). In addition, any high-resolution colour images taken with the pixera can be displayed (Fig. 6a). Finally, the images for a specific well can be displayed next to each other or as a slide show to give a time course for a crystallization experiment (Fig. 6c).

The web interface can be accessed remotely, allowing crystallographers at synchrotrons to plan experimental work in an informed manner with a link back to the crystallization (and protein production and bioinformatics) data. 


\section{conference papers}

\subsection{Crystal handling}

Crystals grown in the 96-well plates need to be handled with extra care, as the small droplets evaporate rapidly once the sealing tape has been removed. Preliminary manipulation is performed without removing the seal by piercing through the tape with a fine needle. Once the seal of a particular well has been removed, crystals need to be mounted in a loop without delay or the drop needs to be enlarged with a stabilizing solution (a $6 \mathrm{~mm}$ thick Perspex plate is used to provide a temporary seal whilst working on a plate). Using the Greiner plate with three platforms per well and dispensing the crystallization droplet only into the middle position has the advantage that the remaining platforms can be used for cryoprotection and soaking experiments. It is important to avoid any change in plate temperature. Plates readily warm up during handling (as apparent from the condensation of reservoir solution onto the sealing tape) and this can damage the crystals throughout the whole plate.

\section{Conclusions}

We report here an overview of the current status of the OPPF crystallization workflow which is used by all groups in the Division of Structural Biology and by a substantial number of outside groups (108 users: 46 from the Division of Structural Biology/OPPF, 50 from elsewhere in the UK and 12 from outside of the UK). To date, over
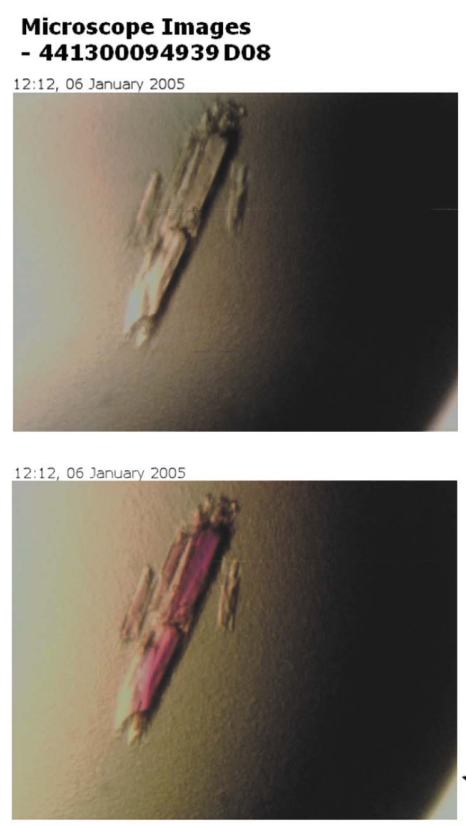

(a)

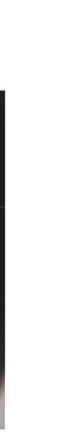

Example Salt Crystals for Block 1 D08

Time Course for 441300094939 Do8
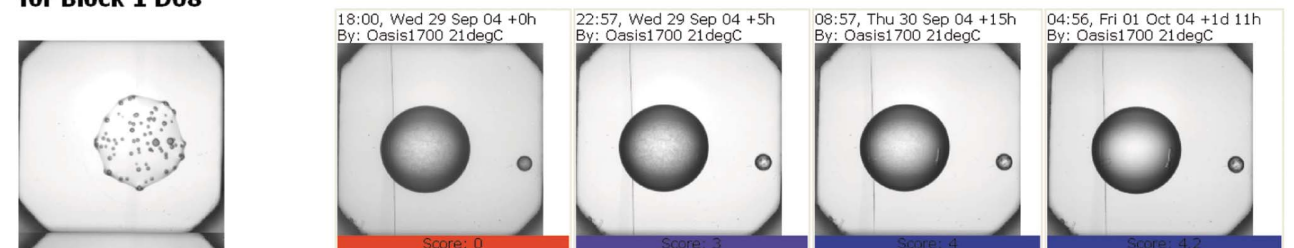

00:56, Sat 02 Oct $04+2 \mathrm{~d}$ 7h 20.57
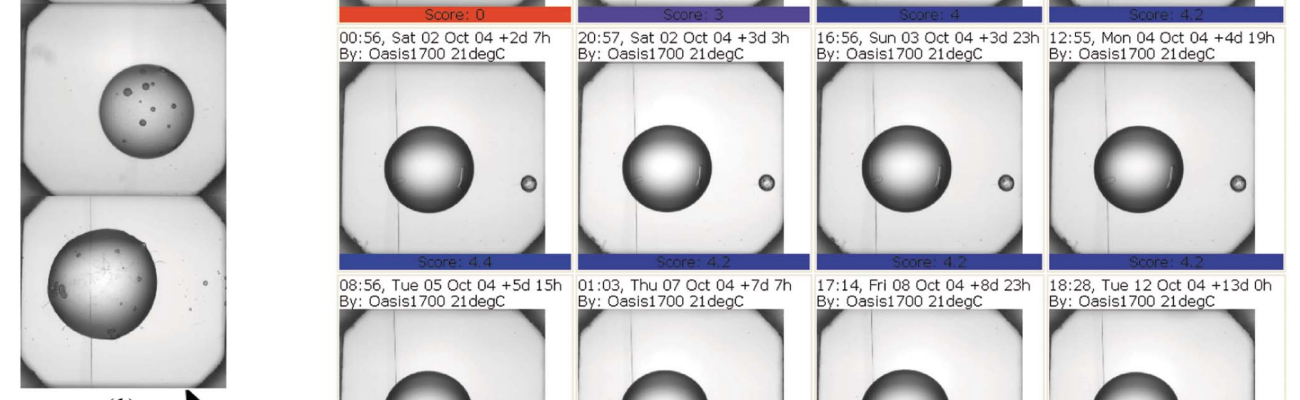

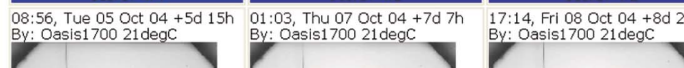

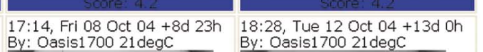
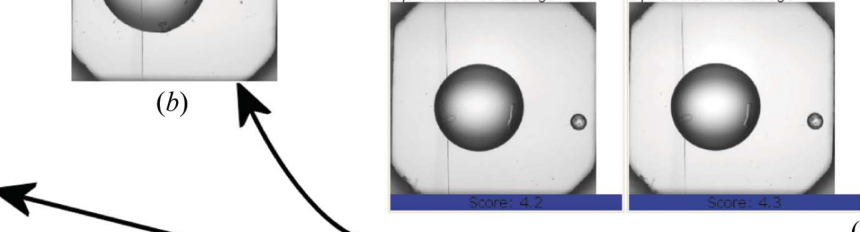

(b)
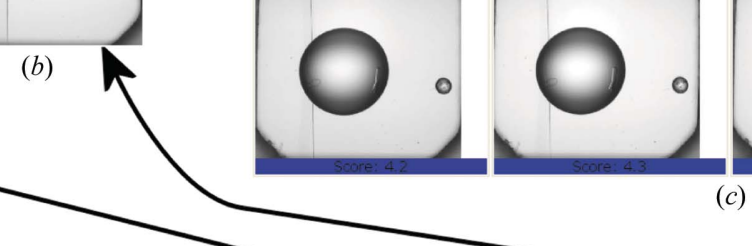

(c)
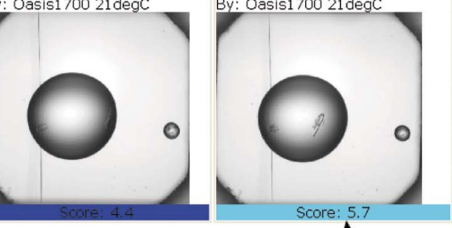

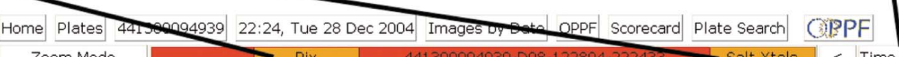

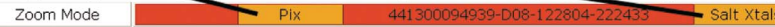

$<\quad 75 \%$

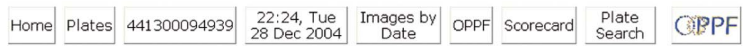

QPPF 44130009493928 December 2004 22:24:17

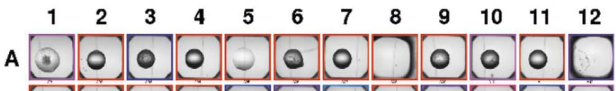

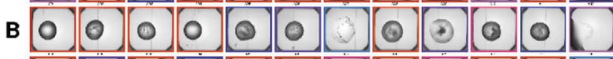

c 0 (0) 000000

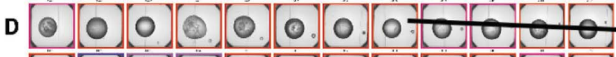

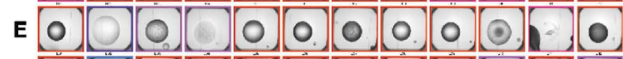

F 0 0 0 o 0 (0) 0 o 0

G 0 (O) 0 (0) 000

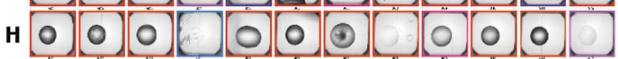

Block 1 - lot\#211079, 211251 - Q 13/9/04

Taken By: Oasis1700 21 degC

Mean Score: 0.88

(d)

Figure 6

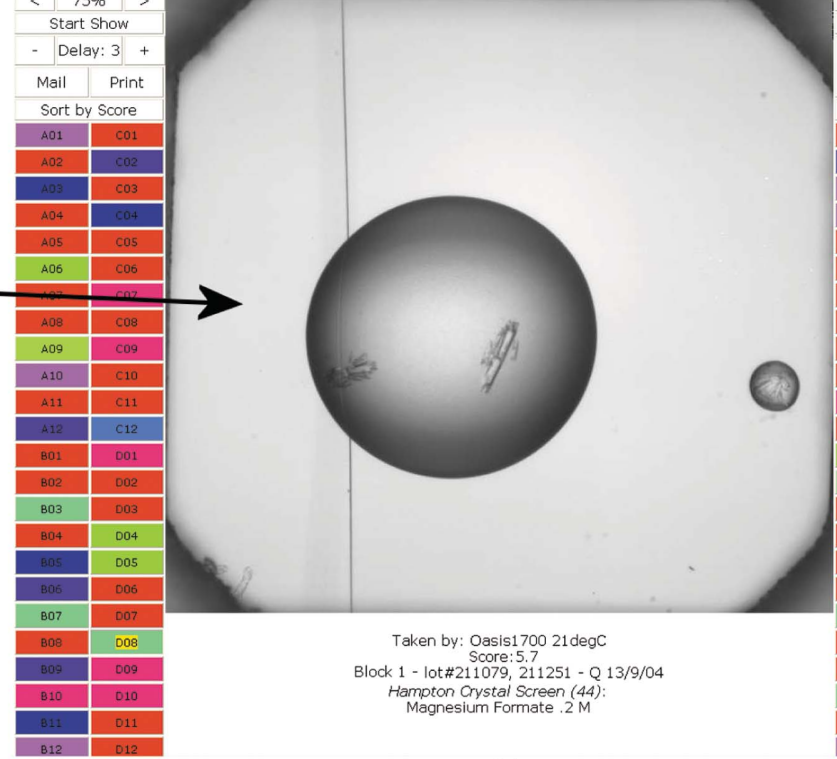

(e)

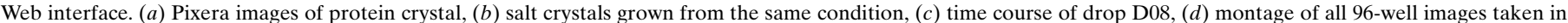
one session, $(e)$ single-well image of drop D8 with user options. Images of protein crystal used with permission (Aricescu et al., unpublished). 
6800 plates have entered the storage vault and over 18 million images of crystallization drops have been automatically captured. These plates represent 592 distinct projects for which 3392 drops have been manually annotated as containing crystals. In total crystals have been reported for 287 projects (including 109 of 215 OPPF specific projects which have entered the vault) and over 70 structures have been determined to date. A more precise indication of the success rate achievable by this technology may be obtained by considering one subproject of the OPPF, namely that addressing structures of a cohort of 48 proteins of Bacillus anthracis. For this cohort 37 proteins have, to date, been expressed and purified and 27 crystallized, a success rate of $73 \%$. Of those proteins crystallized 13 have yielded three-dimensional structures. This level of success in crystallization is similar to that reported for proteins of Thermotoga maritima by the Joint Centre for Structural Genomics (Canaves et al., 2004), although the overall success rate in going from target to structure, at $27 \%$, is rather higher for the OPPF B. anthracis cohort.

Overall the OPPF workflow provides a robust and successful route into protein crystallographic analysis for a very broad range of human, viral and bacterial proteins, both in isolation and as proteinprotein complexes. A simple optimization procedure has been devised which works well and is widely used. We have developed reliable techniques for mounting crystals grown in nanolitre drops directly in cryoloops and now routinely solve structures from such crystals. We are rapidly accumulating a substantial database of crystallization data and images. One major emphasis of current work is to use our library of images to facilitate the development of crystal image-analysis software; this is an area of collaboration with Dr Julie Wilson (Wilson, 2002, 2004). The automated imaging system provides time-course information, which is an extremely powerful aid to crystal identification. A second research area now becoming statistically feasible is data mining crystallization conditions to develop a second generation of improved crystallization screens. The success of our limited protein block (see Fig. 3) demonstrates the power of this approach and a major step in this direction has been taken by the Joint Centre for Structural Genomics (Page \& Stevens, 2004). One possibility is the development of 'bespoke' screens targeted to specific protein classes. We already have evidence of the efficacy of such an approach from a 96-condition screen designed for T-cell receptor and MHC class I molecule crystallization yielding a high success rate for these and related targets.

The Oxford Protein Production Facility is a Medical Research Council-funded pilot project for the UK and is part of the Structural
Proteomics IN Europe (SPINE) consortium (European Commission Grant No. QLG2-CT-2002-00988). CM is funded by the Biotechnology and Biological Science Research Council as part of the e-HTPX grant and IB is funded by the Wellcome Trust (under grant H5RCZRO). JG is a Royal Society University Research Fellow, EYJ is a Cancer Research UK Principal Research Fellow and DIS is a Medical Research Council Research Professor.

\section{References}

Adams, J. A., Jewell, D., Jorgensen, K., Mickley, M. \& Newman, J. M. (2002). $J A L A, 7,36-40$.

Bodenstaff, E. R., Hoedemaeker, F. J., Kuil, M. E., De Vrind, H. P. \& Abrahams, J. P. (2002). Acta Cryst. D58, 1901-1906.

Brown, J. et al. (2003). J. Appl. Cryst. 36, 315-318.

Canaves, J. M., Page, R., Wilson, I. A. \& Stevens, R. C. (2004) J. Mol. Biol. 344, 977-991.

Jaafar, F. M., Attoui, H., Bahar, M. W., Siebold, C., Sutton, G., Mertens, P. P. C., De Micco, P., Stuart, D. I., Grimes, J. M., \& De Lamballerie, X. (2005). Structure, 13, 17-28.

Krupka, H. I., Rupp, B., Segelke, B. W., Lekin, T. P., Wright, D., Wu, H-C., Todd, P. \& Azarani, A. (2002). Acta Cryst. D58, 1523-1526.

Kuhn, P., Wilson, K., Patch, M. G. \& Stevens, R. C. (2002) Curr. Opin. Chem. Biol. 6, 704-710.

Lartigue, A., Gruez, A., Briand, L., Pernollet, J.-C., Spinelli, S., Tegoni, M. \& Cambillau, C. (2003). Acta Cryst. D59, 919-921.

Mayo, C. J., Diprose, J. M., Walter, T. S., Berry, I. M., Wilson, J., Owens, R. J., Jones, E. Y., Harlos, K., Stuart, D. I. \& Esnouf, R. M. (2005). Structure, 13, 175-182.

Mueller, U., Nyarsik, L., Horn, M., Rauth, H., Przewieslik, T., Saenger, W., Lehrach, H. \& Eickhoff, H. (2001). J. Biotechnol. 85, 7-14.

Page, R. \& Stevens, R. C. (2004). Methods, 34, 373-389.

Rose, D. (1999). Drug Discov. Today, 4, 411-419.

Santarsiero, B. D., Yegian, D. T., Lee, C. C., Spraggon, G., Gu, J., Scheibe, D., Uber, D. C., Cornell, E. W., Nordmeyer, R. A., Kolbe, W. F., Jin, J., Jones, A. L., Jaklevic, J. M., Schultz, P. G. \& Stevens, R. C. (2002). J. Appl. Cryst. 35, 278-281.

Shvets, I. V., Makarov, S., Franken, C., Shvets, A., Sweeney, D. \& Osing, J. (2002). JALA, 7, 125-129.

Spraggon, G., Lesley, S. A., Kreusch, A. \& Priestle, J. P. (2002). Acta Cryst. D58, 1915-1923.

Sulzenbacher, G. et al. (2002). Acta Cryst. D58, 2109-2115.

Sutton, G., Fry, E., Carter, L., Sainsbury, S., Walter, T., Nettleship, J., Berrow, N., Owens, R., Gilbert, R., Davidson A., Siddell, S., Poon, L. L. M., Diprose, J. M., Alderton, A., Walsh, M., Grimes, J. M. \& Stuart, D. I. (2004). Structure, 12, 341-353.

Walter, T. S., Diprose, J. M., Brown, J., Pickford, M., Owens, R. J., Stuart, D. I. \& Harlos, K. (2003). J. Appl. Cryst. 36, 308-314.

Wilson, J. (2002). Acta Cryst. D58, 1907-1914.

Wilson, J. (2004). Crystallogr. Rev. 10, 73-84. 